\title{
Forschungspreis
}

\section{Mit „viertem Aggregatzustand“ Keime in chronischen Wunden reduzieren}

— Fest, flüssig, gasförmig - Plasma: Mit dem vierten Aggregatzustand kann die Anzahl von Bakterien auf chronischen Wunden reduziert werden. „Plasma entsteht in einem starken elektrischen Feld und ionisiert dabei das vorherrschende Gas, in unserem Fall Argon", erklärte Dr. Georg Isbary, München, Hauptpreisträger des Forschungspreises 2010 der Fondation URGO, einer gemeinnützigen Stiftung.

Plasma besteht aus verschiedenen aktiven Komponenten - Sauerstoff-, Stickstoff- und Wasserstoffradikalen, aus lonen, Elektronen, Photonen und UV-Strahlung -, die offenbar bakterizid, fungizid und viruzid wirken. Auch ein Effekt auf die Wundheilung durch Einfluss auf Wachstumsfaktoren oder Wundflüssigkeiten wird angenommen. In der Chirurgie und Desinfektion schon länger eingesetzt, gelang es 2003, Plasma unter atmosphärischen Bedingungen kalt zu generieren. Seitdem kann es in medizinischen Verfahren wie der
Wundbehandlung praktisch eingesetzt werden. Dabei wird mit einem speziell entwickelten Gerät, dem MikroPlaSter, das Plasma erzeugt, von $800^{\circ} \mathrm{C}$ auf $23-34^{\circ} \mathrm{C}$ gekühlt und über das Trägergas Argon auf die zu behandelnde Wunde gebracht.

Isbary und Mitarbeiter haben die erste prospektive, randomisierte und kontrollierte klinische Studie mit Niedertemperatur Argon Plasma als Zusatztherapie bei Patienten mit chronischen Wunden durchgeführt. Dabei gelang es, durch eine tägliche fünfminütige Behandlung die Keimzahl signifikant zu reduzieren $\left(34 \%, p<10^{-6}\right)$, unabhängig von der Keimart. Gesundes Gewebe wird nicht geschädigt und die Behandlung gut toleriert. Die Patienten verspüren lediglich einen warmen Lufthauch.

\section{Pfadfinder im Dschungel der Wundbehandlung}

Der zweite Preis ging an Anke Bültemann, Hamburg, und Dr. Gunnar Riepe, Koblenz,

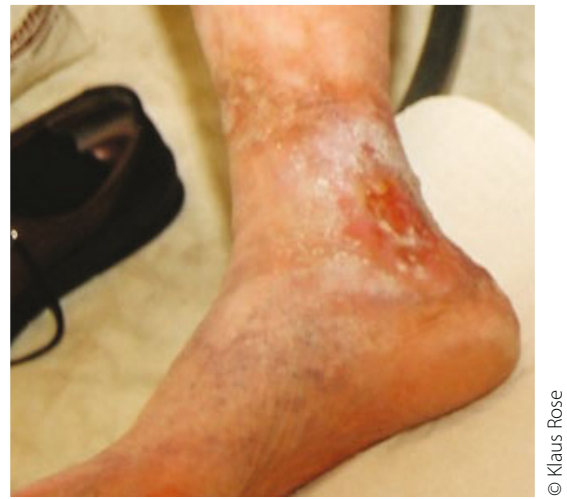

Bei der Argon-Plasma-Zusatztherapie verspürt der Patient nur einen warmen Lufthauch.

für ihre WundUhr. Der „interprofessionelle Pfadfinder im Dschungel der modernen, feuchten, phasengerechten Wundbehandlung" soll die Auswahl des geeigneten Wundprodukts erleichtern, den korrekten und indikationsgerechten Einsatz fördern und damit die Planung, Durchführung und Dokumentation der Behandlung vereinfachen.

- Michael Koczorek

Quelle: Verleihung des Forschungspreises 2010 der Fondation URGO, Deutscher Wundkongress, Bremen, Mai 2011 (Veranstalter: Urgo)

\section{Vorteile der Insulinanaloga nutzen, denn ... \\ „Hypoglykämien sind kein Kavaliersdelikt"}

— Beim Typ-2-Diabetes sollte schon frühzeitig mit einer Insulinbehandlung begonnen werden, um die Nüchternblutzuckerwerte zu normalisieren und "den Patienten den Start in den Tag zu erleichtern". Geeignet ist hierzu aufgrund des geringeren Hypoglykämierisikos vor allem das langwirksame Insulinanalogon Insulindetemir $\left(\right.$ Levemir $\left.^{\circledast}\right)$, sagte Dr. Marcel Kaiser, Frankfurt.

Die Verordnung der modernen Insulinanaloga wird durch das AMNOG unproblematischer, weil nun auch die Herstellerrabatte, die es mit nahezu allen Krankenkassen gibt, bei der Bewertung der Wirtschaftlichkeit berücksichtigt werden. "Das gibt mehr Verordnungssicherheit", erklärte Prof. Bernd Brüggenjürgen, Celle.

Die Patienten können somit weiter von den Vorteilen der modernen Therapie pro- fitieren. Diese sind nach Kaiser vor allem beim Insulindetemir zu realisieren. Unter dem als Basisinsulin zugelassenen Insulinanalogon kommt es Studien zufolge gegenüber NPH-Insulin zu signifikant weniger Hypoglykämien. Die Rate ist bei vergleichbarer $\mathrm{HbA}_{1 c}$-Senkung um $53 \%$ geringer. „Besonders bemerkenswert war die um sogar $65 \%$ geringere Rate nächtlicher Hypoglykämien", betonte der Diabetologe.

Der Unterschied ist relevant, da Hypoglykämien keineswegs „ein Kavaliersdelikt sind". Sie haben erhebliche hämodynamische Auswirkungen, führen zum Pulsanstieg und zum Anstieg des systolischen Blutdrucks sowie der myokardialen Kontraktilität mit erhöhter Ejektionsfraktion. Es kommt ferner zu protrahierten Symptomen wie Cephalgien und Müdigkeit, die auch wirtschaftliche Implikationen haben: vermehrte Fehltage am Arbeitsplatz, allgemeine Produktivitätsminderung sowie zusätzliche Blutzuckermessungen, häufig auch eine Verschlechterung der Blutzuckerkontrolle, weil die Insulindosis zurückgenommen wird. Eine verschlechterte Blutzuckerkontrolle hat im Hinblick auf das Risiko von Folgekomplikationen Bedeutung und damit auch wirtschaftliche Konsequenzen. Der Vermeidung von Hypoglykämien kommt daher, so betonte es auch die Praxis-Leitlinie der DDG, eine wesentliche prognostische Bedeutung zu.

\footnotetext{
- Christine Vetter

Quelle: Media-Lunch „Lang wirksame Insulinanaloga auf dem Prüfstand/Die medizinische Perspektive/Die Kosten-Perspektive", DGIMKongress, Wiesbaden, Mai 2011 (Veranstalter: Novo Nordisk)
} 